\title{
High-fat diets rich in n-3 polyunsaturated fatty acids delay onset of insulin resistance in rats
}

\begin{abstract}
Insulin resistance is a growing worldwide syndrome that predispose human to a number of chronic diseases such as diabetes and cardiovascular diseases. Various studies have indicated that insulin action is highly influenced by diet compositions particularly dietary fat intake and proportion of n-3 and n-6 Polyunsaturated Fatty Acids (PUFA). The current study was designed to assess the influence of High Fat Diet (HFD) with different n-6: n-3 Fatty Acid Ratios (FAR) on insulin sensitivity, plasma triacylglycerol (TAG) and lipoprotein profile. Forty male Sprague-Dawley rats were randomly allocated into four groups and received the isocaloric high fat diets enriched with either high n-6: n-3 fatty acid ratio (HFAR), medium n6: n-3 fatty acid ratio (MFAR), low n-6: n-3 fatty acid ratio (LFAR) and compared with control rats fed standard chow (CTRL). The plasma insulin level of HFAR fed rats manifested significantly $(\mathrm{P}<0.05)$ higher concentration in most of the time points compared to the other groups. The corresponding insulin AUC (ng/L/120min) and insulin sensitivity index of HFAR fed rats appeared to be significantly higher $(\mathrm{P}<0.05)$ than CTRL (335.5 \pm 38.5$)$, LFAR $(273.7 \pm 37.6)$ and MFAR (265.9 \pm 21.7$)$ groups. Blood lipid profile were found to be healthier in the LFAR and MFAR supplemented groups with significantly $(\mathrm{P}<0.05)$ lower total cholesterol and TAG levels. This study showed the possible protective effect against insulin resistance when low n-6: n-3 fatty acid ratio in high fat diets are applied in a rat model.
\end{abstract}

Keyword: N-6: n-3 fatty acid ratio; Insulin sensitivity; Triacylglycerol; Total cholesterol; Insulin; Rat; Chronic diseases 